\title{
Arbitrary Two-Dimensional Multiphoton Excitation Patterns with Temporally Focused Digital Holograms
}

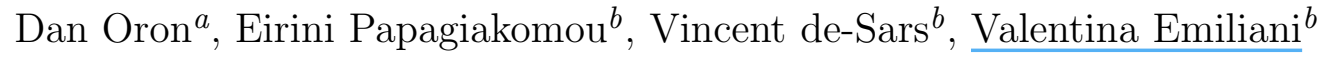 \\ ${ }^{a}$ Weizmann Institute of Science, Department of Physics of Complex System, Rehovot, Israel; \\ ${ }^{b}$ Neurophysiology and New Microscopies Laboratory, Rene Descartes University, Paris, France
}

\begin{abstract}
Multiphoton excitation has recently found application in the fields of bioimaging, uncaging and lithography. In order to fully exploit the advantages of nonlinear excitation, in particular the axial resolution due to nonlinearity, most systems to date operate with point or multipoint excitation, while scanning either the laser beam or the sample to generate the illumination pattern. Here we combine the recently introduced technique of scanningless multiphoton excitation by temporal focusing with recent advances in digital holography to generate arbitrarily shaped, depth resolved, two-dimensional excitation patterns completely without scanning. This is of particular importance in applications requiring uniform excitation of large areas over short time scales, such as neuronal activation by multiphoton uncaging of neurotransmitters. We present an experimental and theoretical analysis of the effect of spatial patterning on the depth resolution achieved in temporal focusing microscopy. It is shown that the depth resolution for holographic excitation is somewhat worse than that achieved for uniform illumination. This is also accompanied by the appearance of a speckle pattern at the temporal focal plane. The origin of the two effects, as well as means to overcome them, are discussed.
\end{abstract}

Keywords: Temporal focusing, Digital holography, Multiphoton microscopy

\section{INTRODUCTION}

Generation of complex three-dimensional optical excitation patterns is a requirement in many practical applications, including optical imaging, uncaging and lithography. Using currently available ultrafast light sources it is simple to localize optical excitation to a point via multiphoton excitation. By scanning this point of excitation (or the excited sample) it is possible to generate arbitrary three-dimensional excitation patterns. Indeed, this is the mode of operation of most multiphoton microscopes ${ }^{1}$ and femtosecond lithography systems ${ }^{2}$ to date. Such sequential point-by point scanning of the excitation is, nevertheless, a slow process, despite many attempts to improve scanning systems, for example by the use of acousto-optic deflectors. ${ }^{3}$ For some applications such as uncaging of biologically active molecules, the scanning process may be too slow relative to the time scale of the probed process. This, combined with the fact that current-day laser sources typically provide orders of magnitude more power than needed for single-point excitation, is the main driving force behind the development of multiple-point excitation systems. ${ }^{4-7}$ Generally, this involves splitting the original beam into several beamlets, each directed to a different point in space. Using beamsplitter arrays, for example ${ }^{6,7}$ this usually results in simple $1 \mathrm{D}$ or $2 \mathrm{D}$ arrays of illumination points.

Recently, the technique of temporal focusing was introduced as a wide-field continuous alternative to these multipoint excitation schemes. ${ }^{8}$ Temporal focusing relies on the utilization of geometrical dispersion to produce a short pulse at the image plane of the objective lens, while the pulse is temporally stretched before and after the focus. This concept is exemplified in Fig. 1. In time-multiplexed multipoint illumination systems the high peak intensity at the focal plane is due to spatial focusing of a pulse with a constant duration, as portrayed in Fig. 1a. In contrast, in temporal focusing (Fig. 1b) the nearly plane wave excitation pulse is split into its frequency components which are then combined in phase to generate a short pulse only at the focal plane. The standard setup for temporal focusing, shown in Fig. 2, includes a blazed reflection grating imaged by a high magnification telescope, comprised of a regular lens and an objective lens, onto the image plane. It

Further author information: (Send correspondence to D.O.)

D.O.: E-mail: dan.oron@weizmann.ac.il, Telephone: 972-8-9346282

Three-Dimensional and Multidimensional Microscopy: Image Acquisition and Processing XVI

edited by Jose-Angel Conchello, Carol J. Cogswell, Tony Wilson, Proc. of SPIE Vol. 7184,

71840S· C 2009 SPIE · CCC code: 1605-7422/09/\$18 · doi: 10.1117/12.808489

Proc. of SPIE Vol. 7184 71840S-1 


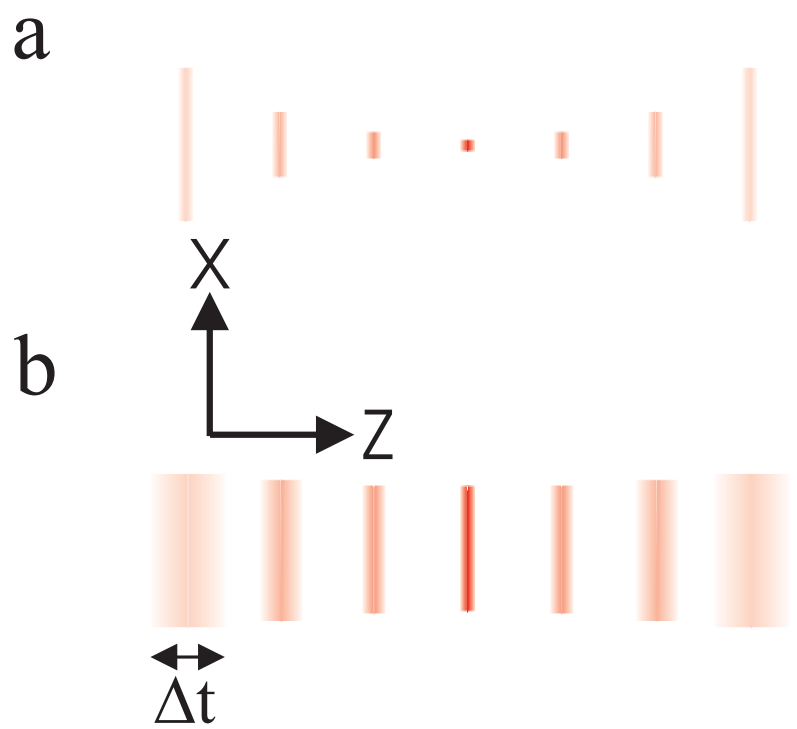

Figure 1. a) Pulse evolution in standard multiphoton imaging based on spatial focusing. The pulse is focused as it propagates through the sample, but its duration remains unchanged. b) Pulse evolution in temporal focusing based multiphoton microscopy. The pulse becomes shorter as it propagates through the sample, reaching its shortest duration at the temporal focal plane, but its spatial profile remains unchanged.

was shown that for a 2D illuminated area the depth resolution afforded by temporal focusing is equivalent to that achieved by line-scanning, while for a $1 \mathrm{D}$ illuminated line (perpendicular to the grating lines), essentially combining temporal focusing with spatial focusing along one spatial dimension, depth resolution is equivalent to that of point scanning. ${ }^{9,10}$ These two cases have a closed-form analytic solution. However, in many practical applications one would like to utilize arbitrary 2D excitation patterns. Since temporal focusing relies on imaging of the grating onto the sample, it is reasonable that spatial patterning of the excitation beam on the grating, as schematically shown in Fig. 2, should result in similarly patterned excitation on the sample, in a similar manner to a mask being imaged onto a sample in optical lithography. The effects of such spatial patterning towards control over the in-plane excitation pattern have not yet been explored. However, it was recently shown that temporal pulse shaping can be used to control the axial response in temporal focusing. ${ }^{11}$

\section{EXPERIMENTAL REALIZATION OF TEMPORALLY FOCUSED DIGITAL HOLOGRAMS}

In attempting to apply temporal focusing with spatially shaped excitation two possible experimental realizations come into mind. the first utilizes amplitude shaping, whereby a spatial amplitude filter is imaged onto the grating surface. While this results in a flat phase front of the excitation pulse, its main drawback is the inefficient utilization of the excitation pulse energy, most of which is blocked. An alternative realization is by the use of Fourier synthesis, whereby a spatial phase filter is Fourier transformed onto the grating surface. Using an appropriate optimization algorithm for the phase pattern (e.g. Gerchberg-Saxton ${ }^{12}$ ), the excitation light is efficiently converted to the required intensity pattern. Here we explore the latter scheme both experimentally and theoretically, determining the effect of holographic illumination on both the excitation pattern and on the axial resolution.

\subsection{Experimental setup}

The experimental realization for the spatiotemporal control of holographic patterning is schematized in Figure 3. As a light source we used a mode-locked Ti:Sapphire laser (Tsunami, Spectra-Physics; $\lambda=780 \mathrm{~nm}, \Delta \lambda=12 \mathrm{~nm}$ ), whose output beam power was controlled by a liquid crystal variable phase-retarder (Meadowlark Optics, LRC200-IR1) combined with a polarizer cube (Meadowlark Optics, BB-050-IR1). The beam was spatially expanded (10x) to match the input window of a LC-SLM (PPM - Programmable Phase Modulator - X8267-13, Hamamatsu 


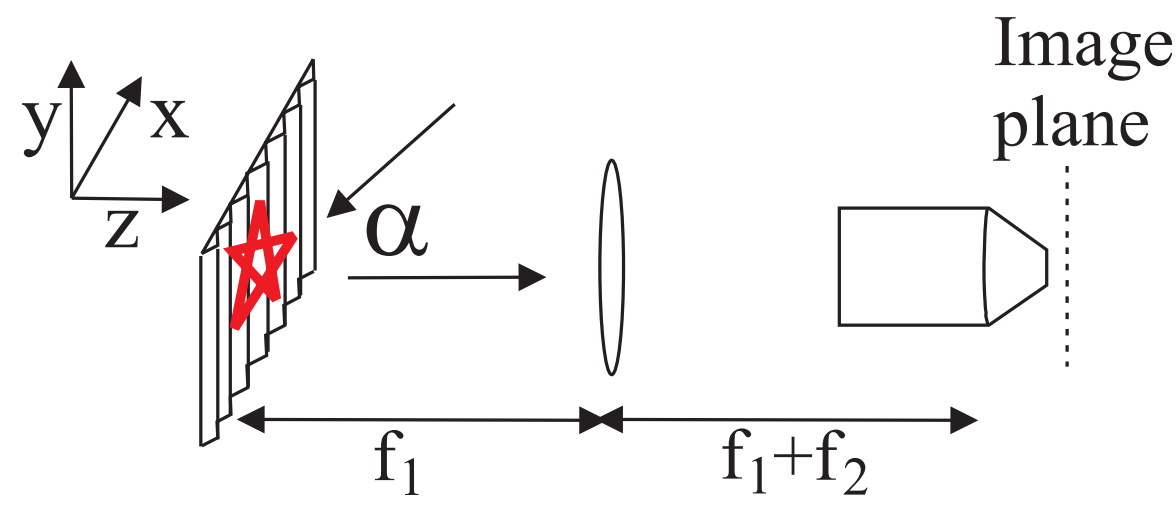

Figure 2. Setup used for temporal focusing. The grating is aligned perpendicular to the optic axis of a high magnification telescope comprised of a lens and a microscope objective. The excitation beam impinges on the grating at an angle $\alpha$ such that the center wavelength is diffracted towards the optic axis. An arbitrary pattern is projected on the grating, either via amplitude or phase-and-amplitude shaping. For the latter, the excitation beam is shaped by a spatial light modulator followed by a Fourier lens.

Photonics K.K.), which operates in reflection mode. The wave front of the beam at the SLM plane was modified by using a custom-designed software that, given a target intensity distribution at the focal plane of the microscope objective, calculates with an iterative Fourier transform algorithm (IFTA $)^{12}$ the corresponding phase-hologram. ${ }^{13}$ After diffraction by the LC-SLM, typically $50 \%$ of the incoming beam goes in the first $(+1)$ order and the rest is distributed among the zero order and higher order components. A phase grating was introduced in phase holograms so as to spatially displace the +1 from the zero order spot. The pulse width after reflection by the SLM was $140 \mathrm{fs}$. The beam reflected from the SLM was Fourier transformed by a $1000 \mathrm{~mm}$ focal length achromatic lens $\left(f_{0}\right)$ and formed a first image of the target intensity onto a blazed reflectance grating $(830 \mathrm{l} / \mathrm{mm})$ placed at the focal plane of L1 and aligned perpendicular to the optical axis of the microscope. An illumination angle of $\alpha=40.5^{\circ}$ was chosen such as the +1 order diffracted beam ( $70 \%$ of the incoming beam) at the center frequency of the excitation pulse was directed along the optical axis of the microscope. The grating dispersed the various frequency components of the pattern, which were imaged onto the sample via a telescope comprised of an achromatic lens $\left(f_{1}=500 \mathrm{~mm}\right.$ focal length) and the microscope objective (Olympus, LUMPLFL60xW/IR2, NA 0.90). This gave rise to a depth-resolved temporally focused excitation pattern at the focal plane of the objective whose lateral spatial distribution was a reduced $(\approx 1 / 170)$ replica of the pattern generated on the grating. Two-photon holographic patterns were used to excite emission from a $0.9 \mu \mathrm{m}$ thick spin-coated fluorescent layer of Rhodamine in PMMA that was collected by a second objective (Olympus, UPLSAPO60xW, NA 1.20) placed opposite to the excitation one. ${ }^{13}$ The use of two independent objectives allowed one to straightforwardly image out of focus planes of the excitation volume and therefore to precisely derive the axial propagation of the excitation beam around the objective focal plane (see below). The fluorescence was imaged by a tube collection lens $\left(f_{t l}=150 \mathrm{~mm}\right)$ to a CCD camera (CoolSNAP HQ2, Roper Scientific). To reject the excitation light, an emission filter (Chroma Technology HQ 535/50M) and a dichroic filter (Chroma Technology 640DCSPXR) were placed in front of the CCD camera.

\subsection{Numerical simulations}

Existing calculations on various effects in temporally focused illumination in the geometry shown in Fig. 2 have generally assumed spatially homogeneous, or at least smooth, illumination patterns. In order to calculate excitation patterns with arbitrary phase- and amplitude- modulated excitation we assume a given complex excitation pattern $A(x, y)$ which is frequency independent impinging upon the grating at an angle such that the central frequency of the pulse is diffracted perpendicular to the grating surface for plane-wave illumination. The excitation pulse spectrum is then discretized, and the propagation from the grating to the image plane is solved separately for each spectral component using the angular spectrum of waves approach, ${ }^{14}$ taking into account the finite aperture of the objective lens. This yields a four-dimensional distribution of complex field amplitudes $A_{\text {image }}(x, y, z, \omega)$. For each point this is Fourier transformed back into time domain to obtain $A_{\text {image }}(x, y, z, t)$, 


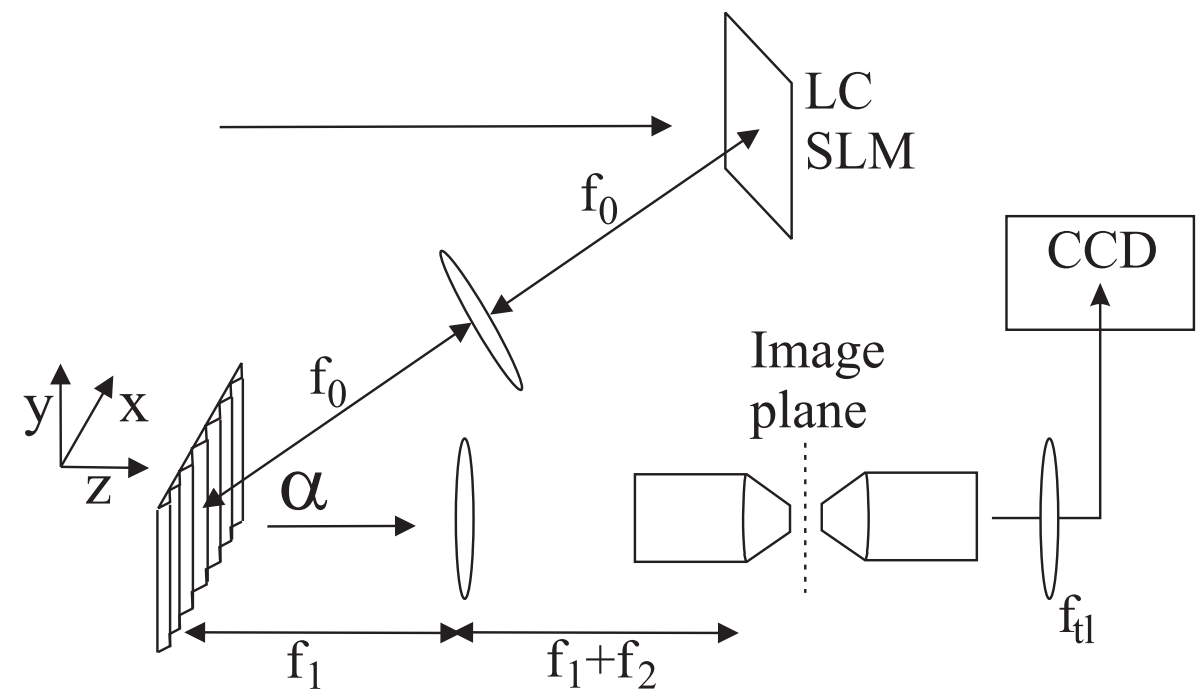

Figure 3. Experimental setup for temporal focusing with holographic beam excitation. The phase pattern imprinted by the SLM is Fourier transformed onto the grating. The two-photon fluorescent emission from the temporal focal plane is imaged onto a CCD camera in transmission mode.

which is then integrated to yield the TPEF intensity according to equation 1. All calculations were scalar, practically assuming a linear polarization of the excitation beam.

$$
I_{T P E F}(x, y, z)=\int_{-\infty}^{\infty} d t\left|A_{\text {image }}(x, y, z, t)\right|^{4}
$$

The calculations described in the following were performed using the experimental conditions described above, and using the following parameterizations: For the 2D case the calculation was performed for a $100 \mu m \mathrm{x} 100 \mu m$ area in the XY plane, where the size of each pixel was approximately $0.25 \mu \mathrm{m}$. Along the propagation direction each pixel was $0.5 \mu \mathrm{m}$. The pulse spectrum, with a FWHM of $8.5 \mathrm{~nm}$, centered at $780 \mathrm{~nm}$, was discretized in the calculation to 61 colors spaced in wavelength by about $0.25 \mathrm{~nm}$.

The results are, of course, quite general in their applicability to other focusing condition, using the simple scaling of temporal focusing with the excitation numerical aperture. ${ }^{10,15}$

\subsection{Experimental results}

In order to test the possibility of generating depth-resolved arbitrary patterns we consider the simple case of excitation of sharply defined circles of several diameters generated by an IFTA algorithm. ${ }^{12}$ The observed excitation patterns in the focal plane, as imaged onto the camera are shown in Fig. 4a. As can be seen, a circular pattern is observed, but it suffers from significant speckle, which is generally not observed in temporal focusing with "smooth" excitation patterns. The appearance of speckle is partly related to the algorithm and to imperfections of the SLM device, but is reinforced by the fact that in the last part of the propagation (from the grating to the objective) a significant portion of the excitation light is diffracted outside the back aperture of the illumination objective. The latter effect is related to rapid phase variations of the illumination beam on the grating, enhancing the formation of dark areas. The relationship between this speckle pattern and the axial resolution will be discussed in the following section.

The axial response of the integrated fluorescence over the entire plane, obtained by moving the relative positions of the excitation objective and the thin fluorescent layer, is shown in Fig. 4b for all three cases. As can be seen, the axial response does not significantly depend on the details of the shape. The observed FWHM of the axial response was $5.5 \pm 0.4 \mu \mathrm{m}$, and the decay at the wings scales as $1 / z$, as is observed in previous realizations of widefield temporal focusing microscopy, having uniform illumination. ${ }^{8}$ The axial resolution is, nevertheless, somewhat worse than in this latter case, where we measured (using the same setup but a uniform illumination 

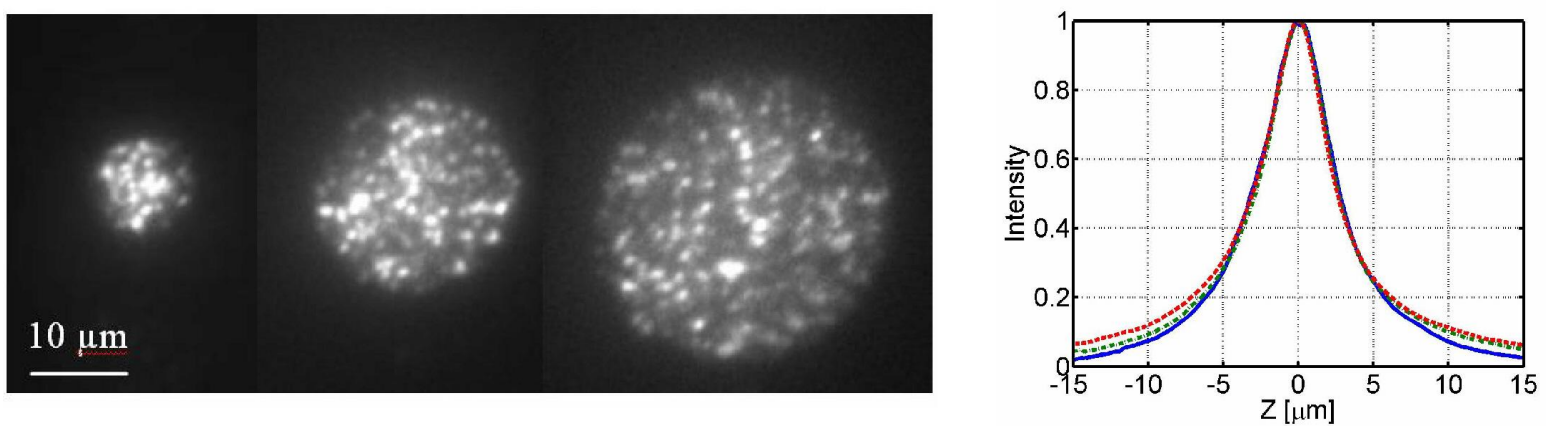

Figure 4. Images of a thin fluorescent layer placed at the temporal focal plane, illuminated by a temporally focused holograms designed to generate full circles of diameters $10 \mu \mathrm{m}, 20 \mu \mathrm{m}, 30 \mu \mathrm{m}$, (left). Z-dependence of the integrated emission for all three cases (solid blue, dashdotted green and dashed red lines, respectively), exhibiting a nearly shapeindependent axial response (right).

on the grating, bypassing both the SLM and the Fourier lens), an axial resolution of $3.2 \mu m$. To account for this, let us compare these results with those obtained from full numerical simulations of phase-modulated excitation patterns.

\subsection{Comparison with simulations}

As discussed previously, determination of the axial response for a phase modulated excitation pulse, as in the case of the excitation profile of a digital hologram, is more complex than that of excitation with slowly varying excitation patterns. Indeed, as long as the phase varies smoothly (that is, there is little phase variation over a distance of the magnified diffraction limited spot) the temporal focusing process should be hardly affected. Rapid phase variations lead, however, to broadening of the distribution of scattering angles for each color. This both interferes with the geometrical dispersion which lies at the basis of the temporal focusing process and results in significant scattering of light outside the back aperture of the objective lens, generating a speckle pattern at the image plane. The limit of pure phase modulation has previously been discussed both theoretically ${ }^{8}$ and experimentally $^{16}$ for the case of head-on excitation through a diffuser (which replaces the grating). It was shown that this results in generation of spatiotemporal speckle at the image plane, stretching, along the propagation direction, to a distance of the order of the spatial extent of the undistorted pulse $c \tau_{\text {pulse }}$ (For high NA excitation). Here we analyze numerically the case of a grating illuminated with a rapidly varying phase pattern. For generality, we show here a calculation for a purely random phase (where the intensity at the grating surface was maintained constant but the phase was randomly chosen between 0 and $2 \pi$ for every region corresponding to a demagnified pixel of $\left.0.25 x 0.25 \mu \mathrm{m}^{2}\right)$.

The calculated two-photon image at the focal plane is shown in Fig. 5a. As can be seen, a speckle pattern with rather isolated peaks is generated. The corresponding $\mathrm{z}$ response curve is shown in Fig. 5b (solid blue line). It shows a $4.5 \mu \mathrm{m}$ FWHM axial response which is broader than the $3.3 \mu \mathrm{m}$ one obtained for a pure intensity modulation (dashed red line). Clearly the case of a purely random phase modulation is an extreme limit of the holographic illumination patterns presented above. Nevertheless, both the speckle pattern and the decrease in the axial resolution appear in good agreement with the experimental results presented in Fig. 4. Moreover, simulations using the initial conditions of the experiments shown in Fig. 4 (not shown here) yield a similar axial response, highlighting the fact that the holographic beams resemble random phase patterns in many aspects. The main difference between purely random phase and the holographic beams is that the fraction of the excitation light which is coupled into the objective is significantly smaller in the former case.

\section{DISCUSSION}

We now consider in more detail the origin of both the appearance of a speckle pattern and of the broadening of the axial response. The speckle pattern has to do with the diffraction efficiency from the grating into the objective back aperture. For a locally "smooth" phase, a case analogous to the standard realization of temporal 

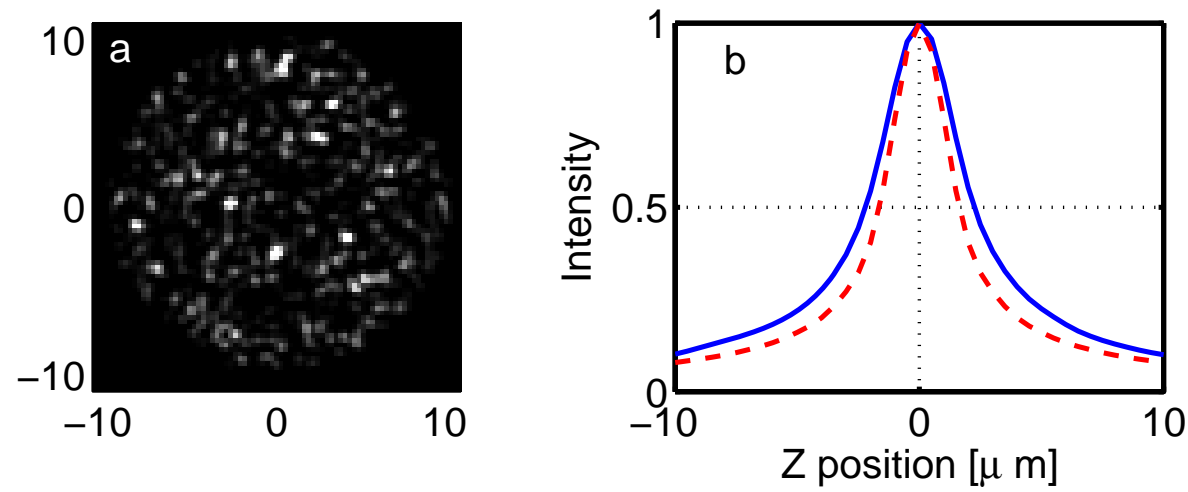

Figure 5. TPEF intensity pattern (a) and calculated depth response (b) for an illumination pattern with random phase. The observed FWHM depth response of $4.5 \mu \mathrm{m}$ (solid blue line) is broader than the $3.3 \mu \mathrm{m}$ FWHM response observed for the case of a constant phase (dashed red line).

focusing, most of the light is diffracted to within the objective back aperture. Similarly, where the phase of the excitation beam varies rapidly (on the scale of the demagnified diffraction limited spot on the grating), much of it will be diffracted outside the objective back aperture. Hence, the observed intensity peaks, corresponding to most of the generated two-photon signal, arise from regions on the grating where the phase pattern is rather smooth. Since locally within these regions the depth resolution comparable with "standard" temporal focusing (corresponding to line scanning) the overall axial response is only slightly broader than in the case of a plane wave impinging on the grating. The small broadening, as observed in Fig. 5b, is due to the presence of some background spatiotemporal speckle (arising from regions with rapid phase variations). ${ }^{16}$

While for some applications the elimination of speckle from the temporally focused image is unnecessary (for example, in uncaging experiments where the diffusion of the uncaged compounds exceeds the spacing between adjacent speckle peaks), in others it may be important. One possible route to this end is to average over many randomized speckle patterns, for example by introducing a rapidly varying random phase pattern (i.e. a rotating diffuser) after the grating. To generate random patterns the diffuser has to be placed at a distance such that the angular scattering of the diffuser effectively shifts the excitation pattern on the grating by more than a diffraction limited spot, corresponding to $L * \theta_{d} \approx M * \lambda / 2 / N A$ (where $\mathrm{M}$ is the temporal focusing telescope magnification), corresponding to several centimeters in our case. An experimental demonstration of this type of smoothing can be found in Papagiakomou et al. ${ }^{17}$

Overall, the combination of temporal focusing with digital holography offers new paths for generation of controlled depth resolved excitation patterns. Where necessary, the speckle patterns appearing in these experiments can be mitigated by averaging (with possible applications in both multiphoton microscopy and ultrafast lithography). The presented scalar theory based on the angular spectrum of waves approach accounts well for the observations in the experiments. We expect that the combination of digital holography with temporal pulse shaping and with polarization modulation can enable further control over the spatial distribution of multiphoton excitation.

\section{REFERENCES}

[1] W. Denk, J.H. Strickler, W.W. Webb, "Two-photon laser scanning fluorescence microscopy," Science 248, $73(1990)$.

[2] R.R. Gattass, E. Mazur, "Femtosecond laser micromachining in transparent materials", Nature Photonics 2, 219 (2008).

[3] X. Lv, C. Zhan, S. Zeng, W.R. Chen, Q. Luo, "Construction of multiphoton laser scanning microscope based on dual-axis acousto-optic deflector", Rev. Sci. Intr. 77, 046101 (2006) and refernces therein; G.D. Reddy, K. Kelleher, R. Fink, P. Saggau, "Three-dimensional random access multiphoton microscopy for functional imaging of neuronal activity", Nature Neuroscience 11, 713 (2008). 
[4] A.H. Buist, M. Muller, J. Squier, G.J. Brakenhoff, "Real-time two-photon absorption microscopy using multipoint excitation," J. Microscopy 192, 217 (1998).

[5] J. Bewersdorf, R. Pick, S.W. Hell, "Multifocal multiphoton microscopy," Opt. Lett. 23, 655 (1998)

[6] D.N. Fittinghoff, P.W. Wiseman, J.A. Squier, "Widefield multiphoton and temporally decorrelated multifocal multiphoton microscopy", Opt. Express 7, 273 (2000)

[7] T. Nielsen, M. Fricke, D. Hellweg, P. Andersen, "High efficiency beam splitter for multifocal multiphoton microscopy," J. Microscopy 201, 368 (2001).

[8] D. Oron, E. Tal, Y. Silberberg, "Scanningless depth resolved microscopy", Opt. Express 13, 1468 (2005).

[9] E. Tal, D. Oron,Y. Silberberg, "Improved depth resolution in video-rate line-scanning multiphoton microscopy using temporal focusing", Opt. Lett. 30, 1686 (2005).

[10] G. Zhu, J. van Howe, M. Durst, W. Zipfel, C. Xu, "Simultaneous spatial and temporal focusing of femtosecond pulses", Opt. Express 13, 2153-2159 (2005).

[11] D. Oron, Y. Silberberg, "Spatiotemporal coherent control using shaped, temporally focused pulses", Opt. Express 13, 9903 (2005); H. Suchowski, D. Oron, Y. Silberberg, "Generation of a dark nonlinear focus", Opt. Comm. 264, 482 (2006).

[12] R.W. Gerchberg, W.O. Saxton, "A pratical algorithm for the determination of the phase from image and diffraction pictures," Optik 35, 237 (1972) ; F. Wyrowski, O. Bryngdahl, "Iterative Fourier-transform algorithm applied to computer holography," J. Opt. Soc. Am. A 5, 1058 (1988).

[13] C. Lutz, T.S. Otis, V. DeSars, S. Charpak, D.A. DiGregorio, V. Emiliani, "Holographic photolysis of caged neurotransmitters," Nat. Methods 5, 821 (2008).

[14] J.W. Goodman, Introduction to Fourier Optics, third edition, (Roberts and Company, Greenwood Village, 2005).

[15] D. Oron, Y. Silberberg, "Harmonic generation with temporally focused ultrashort pulses", J. Opt. Soc. Am. B 22, 2660 (2005).

[16] E. Tal, Y. Silberberg, "Transformation from an ultrashort pulse to a spatiotemporal speckle by a thin scattering surface", Opt. Lett. 31, 3529 (2006).

[17] E. Papagiakoumou, V. de-Sars, D. Oron, V. Emiliani, "Patterned two-photon illumination by spatiotemporal shaping of ultrashort pulses", accepted for publication in Opt. Express (2008). 